\section{NSCLC: EGFR-Exon-20-Insertionen im Visier}

\begin{abstract}
Exon-20-Insertionen (exon20ins) im Gen für den epidermalen Wachstumsfaktorrezeptor (EGFR) finden sich bei $10 \%$ der EGFR-mutierten Adenokarzinome der Lunge. In einer Studie wurde die Prognose von Patienten mit solchen Tumoren unter Therapien mit Tyrosinkinaseinhibitoren (TKI) untersucht.
\end{abstract}

B ei Adenokarzinomen der Lunge mit EGFR-Mutationen hat sich die Therapie mit EGFR-TKI als effizient erwiesen. Als sensitivierende Mutationen gelten z.B. die EGFR-Exon-19-Deletion (exon19del) und die L858R-Punktmutation in Exon 21, die bei einer EGFR-TKITherapie mit einer günstigeren Prognose verbunden sind. Dagegen scheinen Insertionen in Exon 20 eher zur Resistenz gegenüber der Therapie mit TKI zu führen.

Das Team um Jarushka Naidoo analysierte retrospektiv die Daten von 1.882 Patienten mit Adenokarzinomen der Lunge im Stadium IV und nachgewiesener EGFR-exon20ins. Bei 46 (2\%) fand sich diese, 258 (14\%) hatten eine EGFR-
Exon-19-Deletion (exon19del) oder eine L858R-Punktmutation. Die Patienten mit exon20ins waren älter (median 67 Jahre) als jene mit EGFR-exon19del oder L858R (median 63 Jahre; $p=0,01$ ).

Unter den 11 Patienten mit exon20ins, die Erlotinib erhalten hatten, erreichten drei (27\%) ein partielles Ansprechen. Die Zeit bis zur Progression bei TKITherapie betrug bei den Patienten mit EGFR-exon20ins 3 Monate, bei den Patienten mit EGFR-exon19del bzw. L858R 12 Monate $(p<0,01)$. Hingegen war das Ansprechen auf Chemotherapie bei den Patienten mit beiden Mutationstypen ähnlich häufig. Das mediane Gesamtüberleben ab dem Zeitpunkt der Diagnose eines Stadiums IV betrug bei den
Patienten mit exon20ins 26 Monate, bei denen mit exon19del oder L858R $31 \mathrm{Mo-}$ nate $(\mathrm{p}=0,53)$.

Die Ansprechraten bei systemischer Chemotherapie waren bei den Patienten mit EGFR-exon20ins ähnlich hoch wie bei Patienten mit EGFR-mutierten Adenokarzinomen in bislang publizierten Phase-III-Studien.

Fazit: Die Mehrzahl der Lungenkrebspatienten mit Adenokarzinom und EGFRexon20ins spricht auf eine Behandlung mit TKI nicht ausreichend an. In der Erstlinientherapie sei bei diesen Patienten daher eine Standardchemotherapie zu empfehlen, so die Wissenschaftler. $\mathrm{Da}$ verschiedene Insertionsvarianten $\mathrm{zu}$ unterschiedlichen Reaktionen auf TKI führen können, müssten sich weitere Untersuchungen anschließen.

Kathrin von Kieseritzky

Naidoo J et al. Epidermal growth factor receptor exon 20 insertions in advanced lung adenocarcinomas: Clinical outcomes and response to erlotinib. Cancer. 2015;121(18):3212-20.

\section{Neue Kombinationschemotherapie beim NSCLC}

Beim fortgeschrittenen, nichtkleinzelligen Lungenkarzinom (NSCLC) im Stadium III oder IV ist die platinbasierte Chemotherapie Standard. Nun wurde die Kombination Cisplatin plus S-1 mit Cisplatin plus Docetaxel verglichen.

$\mathrm{D}$ as orale Fluoropyrimidin S-1 enthält Tegafur, Gimeracil und Oteracil im Verhältnis 1:0,4:1 und ist als Monotherapie nachweislich gegen NSCLC wirksam. Kaoru Kubota und Kollegen behandelten 608 therapienaive Patienten mit NSCLC im Stadium IIIB oder IV (ECOG-Performancestatus 0-1) aus 66 Kliniken in Japan.

Die Patienten erhielten randomisiert jeweils bis zu 6 Zyklen entweder orales S-1 $\left(80 \mathrm{mg} / \mathrm{m}^{2} / \mathrm{d}\right.$ an den Tagen $\left.1-21\right)$ plus Cisplatin $\left(60 \mathrm{mg} / \mathrm{m}^{2}\right.$ an Tag 8 alle 4-5 Wochen) oder Docetaxel $\left(60 \mathrm{mg} / \mathrm{m}^{2}\right.$ an Tag 1) plus Cisplatin $\left(80 \mathrm{mg} / \mathrm{m}^{2}\right.$ an Tag 1 alle 3-4 Wochen).

Primäres Studienziel war das Gesamtüberleben, sekundäre Endpunkte umfassten das progressionsfreie Überleben (PFS), die Ansprechrate, die Sicherheit und die Lebensqualität.
303 Patienten erhielten S-1 plus Cisplatin, 305 Docetaxel plus Cisplatin. Beim Gesamtüberleben erwies sich die Kombination S-1/Cisplatin der Kombination aus Docetaxel/Cisplatin als nicht unterlegen (medianes Überleben 16,1 vs. 17,1 Monate; Hazard Ratio [HR] 1,013, 96,4\%-Konfidenzintervall [KI] 0,8371,227). Die Forscher fanden auch keine Unterschiede beim PFS: unter S-1/Cisplatin betrug es median 4,9 Monate, unter Docetaxel/Cisplatin median 5,2 Monate (HR 1,113, 95\%-KI 0,945-1,311). Die Gesamtansprechrate lag in der S-1/ Cisplatin-Gruppe bei 26,9\% (komplette Remission [CR]: 1 Patient, partielle Remission [PR]: 77 Patienten), in der Docetaxel/Cisplatin-Gruppe bei 31,3\% (CR: 2 Patienten, PR: 87 Patienten).

Die Krankheitskontrollrate war in beiden Gruppen ähnlich: 74,1\% unter
S-1/Cisplatin, 76,4\% unter Docetaxel/ Cisplatin. In der Docetaxel/CisplatinGruppe wurden im Vergleich zu S-1/Cisplatin signifikant mehr Fälle von febriler Neutropenie (7,4 vs. 1,0\%), Grad-3/4Neutropenien (73,4 vs. 22,9\%), Grad-3/4Infektionen $(14,5$ vs. $5,3 \%)$ und Grad-1/2-Alopezie (59,3 vs. $12,3 \%$ ) registriert. Bezüglich der Lebensqualität zeigten sich im EORTC QLQ-C30- und im LC-13-Fragebogen Vorteile für die Kombination S-1 plus Cisplatin.

Fazit: Die Kombination aus oralem S-1 plus Cisplatin ist der Kombination Docetaxel plus Cisplatin hinsichtlich des Überlebens ebenbürtig und erwies sich bei japanischen Patienten als besser verträglich. Sie könne, so das Résumé der Wissenschaftler um Kubota, als Option in der Erstlinientherapie des fortgeschrittenen NSCLC empfohlen werden.

Kathrin von Kieseritzky

Kubota $\mathrm{K}$ et al. A randomized phase III trial of oral S-1 plus cisplatin versus docetaxel plus cisplatin in Japanese patients with advanced nonsmall-cell lung cancer: TCOG0701 CATS trial. Ann Oncol. 2015;26(7):1401-8. 